\title{
Exploiting Quantization Uncertainty for Enhancing Capacity of Limited-Feedback MISO Ad Hoc Networks
}

\author{
Mohammad G. Khoshkholgh*, A. A. Haghighi ${ }^{@}$, Keivan Navaie ${ }^{\ddagger}$, Kang G. Shin ${ }^{\dagger}$, Victor M. C. Leung* \\ *The University of British Columbia (m.g.khoshkholgh@gmail.com, vleung@ece.ubc.ca) \\ ${ }^{\ddagger}$ Lancaster University (k.navaie@lancaster.ac.uk), ${ }^{\dagger}$ The University of Michigan (kgshin@umich.edu) \\ ${ }^{@}$ ECE, McGill University (asghar.haghighi@gmail.com)
}

\begin{abstract}
In this paper we investigate the capacity of random wireless networks in which transmitters are equipped with multiantennas. A quantized version of channel direction information (CDI) is also available, provided by the associated single antenna receivers. We adopt tools of stochastic geometry and random vector quantization to incorporate the impacts of interference and quantization errors, respectively. We first study the capacity of Aloha, and channel quality information (CQI)-based scheduling, whereby the transmissions decision in each transceiver pair depends on the strength of the CQI against a prescribed threshold. We then propose a new scheduling scheme, namely modified CQI (MCQI), by which the quantization error is effectively incorporated in the scheduling. Further we obtain the capacity of MCQI-based scheduling. Simulation results confirm our analysis and show that the proposed MCQI-based scheduling improves the capacity compared to the CQI-based scheduling and Aloha. It is also seen that the performance boost is more significant where the feedback capacity is low and the network is dense. In comparison with the case of high feedback capacity, the network capacity is not reduced by low feedback capacity in the MCQI-based scheduling. This is of practical importance since the network designer can save the feedback resources by employing MCQI-based scheduling without compromising the capacity and increasing the receivers' complexity.
\end{abstract}

\section{INTRODUCTION}

Infrastructure-less composition of wireless ad hoc communications is practically attractive and has been broadly used in developing technologies, such as device-to-device (D2D) communications [1]. Its lack of centralized coordination however limits the amount of unavoidable signaling overhead. The signaling is required to effectively implement communication protocols, control topology, and manage the interference. It is therefore vital to study the impact of limited signaling overheads on the performance of random networks. In particular for scenarios such as dense networks, essential for the development of Internet of Things, the signalling is required for interference cancellation thus its corresponding overhead is often considered as a limiting factor. In this paper, we investigate the performance of limited-feedback multiantenna ad hoc dense networks.

An MIMO random ad hoc network for very large antenna arrays with no channel state information at the transmitter (CSIT) is considered in [2] where the asymptotic behavior of ergodic rate is investigated. Receivers are however assumed to be capable of completely canceling the impact of a number of strong interferers. Similar approach is considered in other works, see, [3-5]. Nevertheless, such systems suffer from extremely high receiver complexity in addition to overwhelming signaling overheads, making them unfit for the dense networks.

Careful quantization of the required information such as channel direction information (CDI) reduces the signalling overhead. In such cases the receiver often feed its associated transmitter with quantized CDI of the attending channel via the designated feedback channel. The transmitter is then constructing suitable beamforming vectors to reduce the impact of interference inline with the quantized CDI [6]. The importance of quantized beamforming in random networks is investigated in [7] and [8], where significant outage probability improvement is observed comparing to the cases with no CSIT. In [9] net spectral efficiency is introduced as a new metric which sheds light on the design of the feedback capacity in quantized multiple antenna communications. To preserve a required capacity performance, their analysis shows that the optimum feedback capacity is a logarithmic function of the network density.

In such systems, the transmission scheduling is equally important. The current literature mainly focuses on the Aloha scheme, see, e.g, [3], [4], [5], [7], [8], [9], [10], [11] which allows random transmission decision at each individual transceiver pair. Although practically appealing, Aloha technique is unable to incorporate the receiver knowledge of the wireless channel status in the scheduling decision. Therefore, it simply ignores the opportunity to exploit this knowledge to improve the system performance. On that account it is of utmost practical interest to investigate the performance of distributed scheduling schemes in limited-feedback MIMO systems. In this paper, we extend the above analysis by focusing on the impacts of scheduling on the capacity of the quantized beamforming. This, to the best of our knowledge, has not been yet inspected in the related literature.

We study the capacity of channel quality information (CQI) based scheduling and a modified CQI-based scheduling. In the former the CQI of the channel is measured and compared against a threshold, which its value is obtained in our analysis, for deciding upon transmission. In the modified CQI-based scheduling, the knowledge of the receiver about the quantization error of the CDI, which is a function of the angle between 
accurate and quantized CDIs, is exploited to modify the CQI for scheduling purpose. In our analysis, we adopt tools from stochastic geometry [12].

Through analysis we obtained the achievable capacity of a typical transceiver in Aloha, CQI- and MCQI-based scheduling techniques. The analytical results are then utilized to evaluate the scheduling parameters in each case. We further examine the accuracy of our analysis using simulations. and compare the capacity of these three schemes. Simulation results indicate that MCQI-based scheduling outperforms both CQI-based and Aloha. A high capacity improvement is observed for the cases where the network is dense and feedback capacity is small. This is an important result as it enables the network designer to conserve the feedback resources without degrading the capacity performance and imposing extra receiver complexity.

\section{System MOdEL}

We consider an ad hoc communication paradigm in which transmitters are modeled via a Poisson Point Process (PPP) set $\Phi=\left\{X_{i}, i \in \mathbb{N}\right\} \subseteq \mathbb{R}^{2}$, where $X_{i}$ represents the location of transmitter $i$. Each transmitter $X_{i}$ has its associated receiver which is assumed to be located $r$ meters apart. The transmitter nodes are equipped with $N$ transmit antennas while the receivers are single antenna (MISO scenario). The vector channel between each transceiver pair $i$ is denoted by $\boldsymbol{h}_{i}$ where its elements are i.i.d. random variables (r.v.) drawn from complex normal random distribution with zero mean and unit variance. We assume block fading model in which at the start of each time slot the vector fading undergoes a new realization independently and stays fixed during the time slot. Furthermore, we assume $\boldsymbol{h}_{i}$ is independent of $\boldsymbol{h}_{j} \forall i \neq j$.

In this paper, we are interested in examining the performance of scenarios that channel state information (CSI) $\boldsymbol{h}_{i}$ - comprising channel direction information (CDI) $\tilde{\boldsymbol{h}}_{i}=$ $\boldsymbol{h}_{i} /\left\|\boldsymbol{h}_{i}\right\|$ and channel quality information (CQI) $\left\|\boldsymbol{h}_{i}\right\|^{2}-$ is available at the receiver, which is required to be conveyed back to the transmitter through designated limited feedback. As a result, assuming the capacity of feedback channel is $B$ bits, each receiver $i$ quantizes CSI $\tilde{\boldsymbol{h}}_{i}$ into $\hat{\boldsymbol{h}}_{i}$ by applying preconstructed quantization code-book $\mathcal{W}_{B}$, which is available at both transmitter and receiver. The index of the quantized version is eventually sends back to the transmitter. Similar to the previous works, see, e.g., [13]), here we assume that the quantization code-books are constructed by the random vector quantization (RVQ) technique. The feedback channel is also timely and accurate. Further, receiver $i$ has assumed to have access to perfect CSI at the receiver (CSIR). Upon receiving the index, transmitter $i$ extracts $\hat{\boldsymbol{h}}_{i}$ and constructs the beamforming vector, $\hat{\boldsymbol{f}}_{i}=\frac{\hat{\boldsymbol{h}}_{i}}{\left\|\hat{\boldsymbol{h}}_{i}\right\|}$.

We consider the typical transceiver pair with transmitter located at the origin. According to Slyvnyak's Theorem [12][14] it is sufficient to evaluate the performance of the network from the perspective of this typical transceiver with the receiver located at the origin. The experienced Signal-to-Interference ratio (SIR) with CDI quantization is then

$$
\operatorname{SIR}_{0}=\frac{r^{-\alpha}\left\|\boldsymbol{h}_{0}\right\|^{2} \cos ^{2}\left(\theta_{0}\right)}{\sum_{i \in \Phi / X_{0}}\left\|X_{i}\right\|^{-\alpha}\left|\hat{\boldsymbol{f}}_{i}^{\dagger} \boldsymbol{g}_{i}\right|^{2}}
$$

In (1), $\alpha>2$ is the path-loss exponent and $\left\|X_{i}\right\|^{-\alpha}$ is the pathloss attenuation between the transmitter $X_{i}$ and the origin. Without loss of generality we assume that the network is interference-limited thus we ignore the additive white Gaussian noise (AWGN). Finally, $\theta_{i}$ is the phase difference between true and quantized versions of CDI $\tilde{\boldsymbol{h}}_{i}$. Let $\overline{\mathrm{F}}_{\sin ^{2}\left(\theta_{i}\right)}(z)$ be the Complementary Cumulative Distribution Function (CCDF) of quantization error, $Z_{i}=\sin ^{2}\left(\theta_{i}\right)$. For the case of RVQ

$$
\bar{F}_{Z_{i}}(z)=\left(1-z^{N-1}\right)^{2^{B}}, 0 \leq z \leq 1
$$

where $Z_{i}$ s are i.i.d.

Here our main objective is to evaluate the achievable capacity of the typical communication link defined as

$$
\bar{R}=\mathbb{E} \log \left(1+\mathrm{SIR}_{0}\right)
$$

In (3) expectation operator $\mathbb{E}$ acts on all random parameters including the position of transmitters, fading, and quantized beamforming vectors.

To evaluate (3) we need to specify the corresponding scheduling method. In this paper we chiefly focus on the following scheduling schemes: $(i)$ Aloha scheduling whereby in each time slot each transmitter $X_{i}$ randomly turns on with probability $p$; (ii) CQI-based scheduling which depends on the strength of the channel quality indicator (CQI) at the receiver $i$; and (iii) Modified CQI-based scheduling which is similar to CQI where the quantization error is taken into account in the the scheduling decision made in each transmitter.

\section{PERformance AnAlysis}

\section{A. Aloha}

Let assume each node $i$ decides to transmit with probability $p \in[0,1]$ (activity factor). The following proposition provides the capacity of Aloha technique.

Proposition 1: For a given activity factor, $p$, and feedback capacity, $B$ bits, the achievable capacity of each node is

$$
R_{\mathrm{A}}=\int_{0}^{\infty} \frac{\left.p\left(1-\mathbb{E}_{Z}\left(1+w r^{-\alpha}(1-Z)\right)\right)^{-N}\right)}{w e^{p \lambda w^{\check{\alpha}} C(\alpha)}} d w
$$

where $C(\alpha)=\pi \Gamma(1-\check{\alpha}) \Gamma(1+\check{\alpha})$, and $Z$ is the quantization error.

Proof: Let $Z_{0}=\sin ^{2}\left(\theta_{0}\right)$ be the quantization error associated with transceiver pair 0 . Using the results in [9] and [15],

$$
\begin{aligned}
& R_{\mathrm{A}}=p \mathbb{E} \int_{0}^{\infty} \frac{e^{-w}}{w}\left(1-e^{-w \frac{r^{-\alpha}\left\|\boldsymbol{h}_{o}\right\|^{2} \cos ^{2}\left(\theta_{o}\right)}{\sum_{i \in \Phi_{t}}\left\|X_{i}\right\|^{-\alpha}\left|\hat{\boldsymbol{f}}_{i}^{\dagger} \boldsymbol{g}_{i}\right|^{2}}}\right) d w \\
& =p \int_{0}^{\infty} \frac{1}{w} \mathbb{E} e^{-w \sum_{i \in \Phi_{t}}\left\|X_{i}\right\|^{-\alpha} G_{i}}\left(1-\mathbb{E} e^{-w r^{-\alpha} H_{0}\left(1-Z_{0}\right)}\right) d w,
\end{aligned}
$$


where $H_{0}=\left\|\boldsymbol{h}_{o}\right\|^{2}$ is a Chi-squared r.v. with $2 N$ degreesof-freedom (DoF) and is independent of $Z_{0}$. In (5) $\Phi_{t}$ is the set of active transmitters and is a PPP with density of $p \lambda$. To obtain (5) we have simply apply a change of variable and independencies of the involved random variables in the SIR (1). In (1), random variable $G_{i}=\left|\hat{\boldsymbol{f}}_{i}^{\dagger} \boldsymbol{g}_{i}\right|^{2}$ is exponentially distributed (see, [5]) because each receiver $i$ independently quantizes the associated CDI and the beamforming vector $\hat{\boldsymbol{f}}_{i}$ is merely constructed based on the quantized CDI $\hat{\boldsymbol{h}}_{i}$. Using the above facts, and following the results of [14] we can show that for a given $w$

$$
\mathbb{E} e^{-w \sum_{i \in \Phi_{t}}\left\|X_{i}\right\|^{-\alpha} G_{i}}=e^{-p \lambda C(\alpha) w^{\check{\alpha}}} .
$$

$H_{0}$ is a Chi-squared r.v. with DoF of $2 N$ and independent of $Z_{0}$, therefore

$$
\left.\mathbb{E} e^{-w r^{-\alpha} H_{0}\left(1-Z_{0}\right)}=\mathbb{E}_{Z}\left(1+w r^{-\alpha}(1-Z)\right)\right)^{-N} .
$$

Substituting (8) and (7) into (5) completes the proof.

For the particular case of RVQ (see (2)), using straight forward derivations, we can show that for specific value $w$ expression (7) is

$$
\begin{gathered}
(7)=\frac{N-1}{2^{-B}} \sum_{l=0}^{2^{B}-1} \sum_{n=0}^{l(N-1)+N-2} \sum_{m=0}^{n}\left(\begin{array}{c}
2^{B}-1 \\
l
\end{array}\right)\left(\begin{array}{c}
n \\
m
\end{array}\right) \\
\times\left(\begin{array}{c}
l(N-1)+N-2 \\
n
\end{array}\right) \frac{(-1)^{l+n}\left(\left(1+\frac{w}{r^{\alpha}}\right)^{m-N+1}-1\right)}{\left(\frac{w}{r^{\alpha}}\right)^{n+1}(m-N+1)} .
\end{gathered}
$$

Substituting (8) into (4), the capacity is

$$
\begin{gathered}
R_{\mathrm{A}}=p \frac{N-1}{2^{-B}} \sum_{l=1}^{2^{B}-1} \sum_{n=0}^{l(N-1)+N-2} \sum_{m=0}^{n}\left(\begin{array}{c}
2^{B}-1 \\
l
\end{array}\right)\left(\begin{array}{c}
n \\
m
\end{array}\right) \\
\frac{\left(\begin{array}{c}
l(N-1)+N-2 \\
n
\end{array}\right)(-1)^{l+n+1}}{r^{-(n+1) \alpha}(m-N+1)} \int_{0}^{\infty} \frac{\left(\left(1+\frac{w}{r^{\alpha}}\right)^{m-N+1}-1\right)}{w^{n \alpha+1} e^{p \lambda C(\alpha) w^{\check{\alpha}}}} d w .
\end{gathered}
$$

Using Jensen's inequality the following upper-bound is obtained on the capacity:

$$
\begin{gathered}
R_{\mathrm{A}}^{u}=p \log \left(1+\mathbb{E} \operatorname{SIR}_{o}\right) \\
=p \log \left(1+r^{-\alpha} \mathbb{E}\left[H_{0}\right] \mathbb{E}\left[1-Z_{0}\right] \mathbb{E} \frac{1}{\sum_{i \in \Phi} P_{i}\left\|X_{i}\right\|^{-\alpha} G_{i}}\right) \\
=p \log \left(1+N r^{-\alpha}(1-\tilde{\delta}) \int_{0}^{\infty} \mathbb{E} e^{-t \sum_{i \in \Phi_{t}} P_{i}\left\|X_{i}\right\|^{-\alpha} G_{i}} d t\right) \\
=p \log \left(1+r^{-\alpha} N(1-\tilde{\delta}) \int_{0}^{\infty} e^{-p \lambda t^{\check{\alpha}} C(\alpha)} d t\right) \\
=p \log \left(1+p^{-\frac{\alpha}{2}} \hat{C}(\alpha)\right),
\end{gathered}
$$

where due to RVQ assumption, $\tilde{\delta}=2^{B} \Gamma\left(2^{B}, \frac{N}{N-1}\right)$, and

$$
\hat{C}(\alpha) \triangleq \frac{N(1-\tilde{\delta}) \Gamma\left(\frac{\alpha}{2}+1\right)}{r^{\alpha}(\lambda C(\alpha))^{\frac{\alpha}{2}}} .
$$

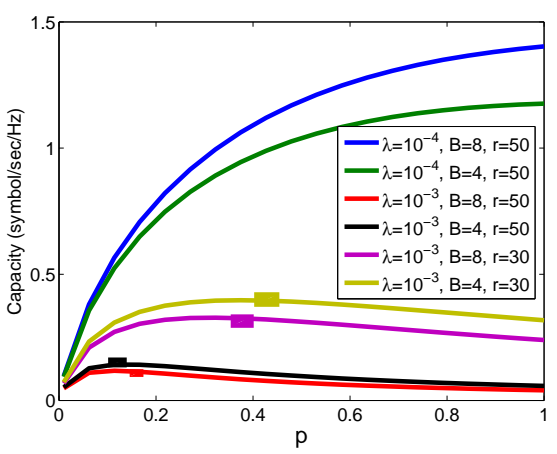

Fig. 1. Achievable capacity of CQI-based scheduling vs. $p$ for $\alpha=4$ and $N=5$.

Using (10) we are then able to find the optimal activity factor, $p$, which results in the maximum upper-bound capacity (10). Straightforward mathematics derivations reveal that this activity factor is obtained by solving the following equation

$$
p^{\frac{\alpha}{2}}\left(1-e^{-\frac{\frac{\alpha}{2} p^{1-\frac{\alpha}{2}} \hat{C}(\alpha)}{1+\hat{C}(\alpha) p^{\frac{\alpha}{2}}}}\right)=\hat{C}(\alpha) .
$$

In Fig. 1 we present a plot of the capacity (9) for several values of $B$ and $\lambda v s$. $p$. The values obtained from (12) are indicated in Fig. 1 by small squares. As it is seen, solutions of (12) are adequately accurate for providing estimation of the best activity factor. Fig. 1 also shows that the capacity is very sensitive to the density of the transmitters and for small $\lambda$, activity factor 1 is optimal. It is also seen that for this case reducing $B$ decreases the capacity and the amount of decrease is higher for larger activity factors. For large values of $\lambda$, the available feedback capacity, $B$, has a smaller impact on the achievable capacity as very high interference overwhelms the achievable capacity.

\section{B. CQI-Based Scheduling}

Aloha scheme is simple however it overlooks the receivers on the intended channel state. This knowledge is exploited could be considered as a base for scheduling. Here, we utilize CQI of the channel, $\left\|\boldsymbol{h}_{i}\right\|^{2}$, to decide upon the transmission status. Similar approach is considered in [11] for outage probability evaluation in single-antenna ad hoc communication systems.

By introducing a threshold, $\gamma_{t h}$, we propose the following CQI-based scheduling. Let $P_{i}$ be the transmission decision at transmitter node $X_{i}$, thus

$$
P_{i}=1\left(r^{-\alpha}\left\|\boldsymbol{h}_{i}\right\|^{2} \geq \gamma_{t h}\right) .
$$

The following proposition provides the achievable capacity of CQI-based scheduling.

Proposition 2: In the CQI-based scheduling with parameter $\gamma_{t h}$ and feedback capacity $B$ bits, the achievable capacity is

$$
R_{\mathrm{C}}=\int_{0}^{\infty} \frac{\left(p_{\mathrm{C}}-\mathbb{E}_{Z} \frac{\gamma_{u}\left(N, \gamma_{t h} r^{\alpha}+w \gamma_{t h}(1-Z)\right)}{\left(1+w r^{-\alpha}(1-Z)\right)^{N}}\right)}{w e^{p_{\mathrm{C}} \lambda w^{\check{\alpha}} C(\alpha)}} d w,
$$


where $\gamma_{u}(N, x)=\int_{x}^{\infty} \frac{t^{N-1} e^{-t}}{\Gamma(N)} d t$, and $p_{\mathrm{C}}$ is the probability that the typical transceiver decides to transmit:

$$
p_{\mathrm{C}}=\overline{\mathrm{F}}_{\|\boldsymbol{h}\|^{2}}\left(\gamma_{t h} r^{\alpha}\right)=e^{-\gamma_{t h} r^{\alpha}} \sum_{n=0}^{N-1} \frac{\left(\gamma_{t h} r^{\alpha}\right)^{n}}{n !},
$$

Proof: Following the same lines of argument as in the proof of Proposition 1, we see that

$R_{\mathrm{C}}=p_{\mathrm{C}} \int_{0}^{\infty} \frac{\left(1-\mathbb{E}\left[e^{-w r^{-\alpha}\left\|\boldsymbol{h}_{o}\right\|^{2}\left(1-Z_{0}\right)} \mid\left\|\boldsymbol{h}_{o}\right\|^{2} \geq \gamma_{t h} r^{\alpha}\right]\right)}{w e^{p_{\mathrm{C}} \lambda w^{\check{\alpha}} C(\alpha)}} d w$,

where $p_{\mathrm{C}}$ is given in (15). We then note that $\mathbb{E}[X]=$ $\int_{t>0} \mathbb{P}\{X>t\} d t$, thus

$$
\begin{gathered}
\mathbb{E}\left[e^{-w r^{-\alpha}\left\|\boldsymbol{h}_{o}\right\|^{2}\left(1-Z_{0}\right)} \mid\left\|\boldsymbol{h}_{o}\right\|^{2} \geq \gamma_{t h} r^{\alpha}\right] \\
=\int_{0<t \leq 1} \mathbb{P}\left\{e^{-w r^{-\alpha}\left\|\boldsymbol{h}_{o}\right\|^{2}\left(1-Z_{0}\right)} \mid\left\|\boldsymbol{h}_{o}\right\|^{2} \geq \gamma_{t h} r^{\alpha}\right\} d t \\
=\frac{1}{p_{\mathrm{C}}} \mathbb{E}_{Z_{0}} \int_{0<t \leq e^{-\gamma_{t h} w\left(1-Z_{0}\right)}} \mathbb{P}\left\{\gamma_{t h} r^{\alpha} \leq\left\|\boldsymbol{h}_{o}\right\|^{2} \leq \frac{r^{\alpha} \log \frac{1}{t}}{w\left(1-Z_{0}\right)}\right\} d t \\
=\mathbb{E}_{Z_{0}} \frac{w\left(1-Z_{0}\right)}{p_{\mathrm{C}}} \int_{\gamma_{t h}}^{\infty} \mathbb{P}\left\{\gamma_{t h} r^{\alpha} \leq\left\|\boldsymbol{h}_{o}\right\|^{2} \leq r^{\alpha} v\right\} e^{-v w\left(1-Z_{0}\right)} d v \\
=\mathbb{E}_{Z_{0}} \frac{w\left(1-Z_{0}\right)}{\Gamma(N) p_{\mathrm{C}}} \int_{\gamma_{t h} \gamma_{t h} r^{\alpha}}^{\infty} e^{-h} h^{N-1} e^{-v w\left(1-Z_{0}\right)} d h d v \\
=\frac{1}{p_{\mathrm{C}}} \mathbb{E}_{Z_{0}} \int_{\gamma_{t h} r^{\alpha}}^{\infty} e^{-w r^{-\alpha} h\left(1-Z_{0}\right)} \mathrm{f}_{\|\boldsymbol{h}\|^{2}}(h) d h \\
=\frac{1}{p_{\mathrm{C}}} \mathbb{E}_{Z_{0}} \frac{\gamma_{t h} r^{\alpha}+w \gamma_{t h}\left(1-Z_{0}\right)}{\left(1+w r^{-\alpha}\left(1-Z_{0}\right)\right)^{N}} \\
\mathbb{E}_{Z_{0}} \frac{\gamma_{u}\left(N \|^{2}(h) d h\right.}{\left(1+\gamma_{t h} r^{\alpha}+w \gamma_{t h}\left(1-Z_{0}\right)\right)}
\end{gathered}
$$

Substituting this in (17) completes the proof.

To estimate the best threshold $\gamma_{t h}$, we use an approximation of the achievable capacity in (14). Applying Jensen's inequality to (14) following by some straightforward mathematical manipulations an upper-bound on the achievable capacity is obtained as the following:

$$
R_{\mathrm{C}}^{u}=p_{\mathrm{C}} \log \left(1+\frac{\gamma_{u}\left(N+1, \gamma_{t h} r^{\alpha}\right)}{p_{\mathrm{C}}^{\frac{\alpha}{2}+1}} \hat{C}(\alpha)\right) .
$$

where $\hat{C}(\alpha)$ is defined in (11).

Let $\dot{p}_{\mathrm{C}}=r^{\alpha} \mathrm{f}_{\|\boldsymbol{h}\|^{2}}\left(\gamma_{t h} r^{\alpha}\right)$ be the derivative of $p_{\mathrm{C}}$ in (15) with respect to $\gamma_{t h}$. By taking derivative of (10) with respect to $\gamma_{t h}$, and setting the resultant to zero, it is then possible to obtain an approximate of the best threshold value that results in the maximum achievable capacity. Doing so, an approximate

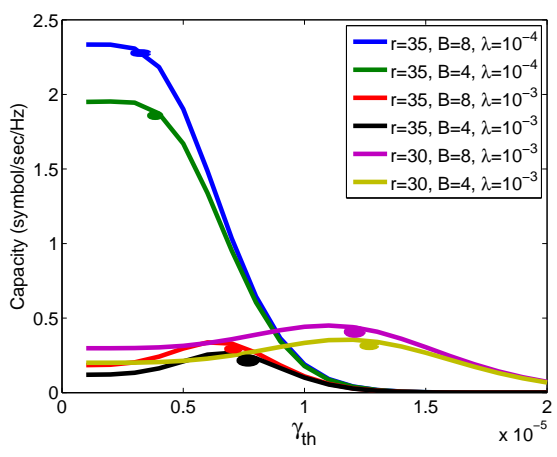

Fig. 2. Achievable capacity of the CQI-based scheduling vs. $\gamma_{t h}$ for $\alpha=4$ and $N=5$.

of the optimal threshold value is obtained by solving the following equation for $\gamma_{t h}$ :

$$
\begin{gathered}
\log \left(1+\frac{\gamma_{u}\left(N+1, \gamma_{t h} r^{\alpha}\right)}{p_{\mathrm{C}}^{\frac{\alpha}{2}+1}} \hat{C}(\alpha)\right) \\
=\hat{C}(\alpha) \frac{\left(\frac{\alpha}{2}+1\right) \gamma_{u}\left(N+1, \gamma_{t h} r^{\alpha}\right)+N p_{\mathrm{C}} \gamma_{t h} r^{\alpha}}{p_{\mathrm{C}}^{\frac{\alpha}{2}+1}+\gamma_{u}\left(N+1, \gamma_{t h} r^{\alpha}\right) \hat{C}(\alpha)} .
\end{gathered}
$$

In Fig. 2 the achievable capacity performance in (14) is given vs. $\gamma_{t h}$ for several values of density and feedback capacity. The obtained estimation of $\gamma_{t h}$ according to equation (19) is also given in Fig. 2 which is seen to be accurate enough to predict the best threshold value yielding the maximum capacity.

It is also seen in Fig. 2 that by increasing the density, the capacity is substantially reduced. However, even if density is very small, using a $\gamma_{t h}$ larger than the best prescribed value, the capacity might become even smaller that the case with a high density. Fig. 2 also indicates that the achievable capacity is more sensitive to the feedback capacity in low density networks, i.e., smaller values of $\lambda$, comparing to the high density scenarios.

\section{MCQI-Based Scheduling}

In CQI-based scheduling, the CQI of the channel, $\boldsymbol{h}_{i}$, is utilized for scheduling. However, in reality the strength of the received signal, as it is also seen in (1) depends on the quantization error through $1-\sin ^{2}\left(\theta_{i}\right)$. In this section we introduce a new scheduling scheme, called modified-CQI (MCQI) scheduling which incorporates the quantization error in the scheduling. Let $P_{i}$ be the transmission decision at the node $i$, where

$$
P_{i}=1\left(\left\|\boldsymbol{h}_{i}\right\|^{2}\left(1-\sin ^{2}\left(\theta_{i}\right)\right) \geq \gamma_{t h} r^{\alpha}\right) .
$$

It is important to note that at the start of each time slot the modified CQI is measurable at each receiver. Quantizing CDI, $\tilde{\boldsymbol{h}}_{i}$, into a quantized index $l$, receiver $i$ can then calculate quantization error by calculating the square of absolute value of the inner product of $\tilde{\boldsymbol{h}}_{i}$ and conjugate of $l$-th code-word, see, e.g., [13]. The achieved capacity of MCQI is given in Proposition 3. 
Proposition 3: In MCQI-based scheduling with parameter $\gamma_{t h}$ and feedback capacity $B$ bits, the achievable capacity is

$$
R_{\mathrm{M}}=\int_{0}^{\infty} \frac{\left(p_{\mathrm{M}}-\mathbb{E}_{Z} \frac{\gamma_{u}\left(N, \frac{\gamma_{t h} r^{\alpha}}{1-Z}+w \gamma_{t h}\right)}{\left(1+w r^{-\alpha}(1-Z)\right)^{N}}\right)}{w e^{p_{\mathrm{M}} \lambda w^{\check{\alpha}} C(\alpha)}} d w,
$$

where $p_{\mathrm{M}}$ is the probability that the typical transceiver decides to transmit:

$$
p_{\mathrm{M}}=\sum_{n=0}^{N-1} \frac{\left(\gamma_{t h} r^{\alpha}\right)^{n}}{n !} \mathbb{E}_{Z}\left[\frac{e^{-\frac{\gamma_{t h} r^{\alpha}}{1-Z}}}{(1-Z)^{n}}\right]
$$

Proof: Starting from the results in [15] we write

$$
R_{\mathrm{M}}=p_{\mathrm{M}} \int_{0}^{\infty} \frac{\left(1-\mathbb{E}\left[e^{-\frac{w}{r^{\alpha}}\left\|\boldsymbol{h}_{o}\right\|^{2}\left(1-Z_{0}\right)} \mid\left\|\boldsymbol{h}_{o}\right\|^{2} \geq \frac{\gamma_{t h} r^{\alpha}}{\left(1-Z_{0}\right)}\right]\right)}{w e^{p_{\mathrm{M}} \lambda w^{\check{\alpha}} C(\alpha)}} d w,
$$

where $p_{\mathrm{M}}$ is given in (22). Further,

$$
\begin{aligned}
& \mathbb{E}\left[e^{-\frac{w}{r^{\alpha}}\left\|\boldsymbol{h}_{o}\right\|^{2}\left(1-Z_{0}\right)} \mid\left\|\boldsymbol{h}_{o}\right\|^{2}\left(1-Z_{0}\right) \geq \gamma_{t h} r^{\alpha}\right] \\
& =\frac{1}{p_{\mathrm{M}}} \mathbb{E}_{Z_{0}} \int_{0}^{e^{-\gamma_{t h} w}} \mathbb{P}\left\{\gamma_{t h} r^{\alpha} \leq\left\|\boldsymbol{h}_{o}\right\|^{2}\left(1-Z_{0}\right) \leq \frac{r^{\alpha} \log \frac{1}{t}}{w}\right\} d t \\
& =\frac{w}{p_{\mathrm{M}}} \mathbb{E}_{Z_{0}} \int_{\gamma_{t h}}^{\infty} \mathbb{P}\left\{\gamma_{t h} r^{\alpha} \leq\left\|\boldsymbol{h}_{o}\right\|^{2}\left(1-Z_{0}\right) \leq r^{\alpha} v\right\} e^{-v w} d v \\
& =\frac{w}{p_{\mathrm{M}}} \mathbb{E}_{Z_{0}} \int_{\gamma_{t h}}^{\infty} \int_{\gamma_{t h} r^{\alpha}}^{r^{\alpha} v} \mathrm{f}_{\|\boldsymbol{h}\|^{2}\left(1-Z_{0}\right)}(v) e^{-v w} d h d v \\
& =\frac{1}{p_{\mathrm{M}}} \int_{\gamma_{t h} r^{\alpha}}^{\infty} e^{-w r^{-\alpha} v} \mathrm{f}_{\|\boldsymbol{h}\|^{2}\left(1-Z_{0}\right)}(v) d v \\
& =\frac{1}{p_{\mathrm{M}}} \int_{\gamma_{t h} r^{\alpha}}^{\infty} e^{-w r^{-\alpha} v} \mathbb{E}_{Z_{0}} \frac{1}{1-Z_{0}} \mathrm{f}_{\|\boldsymbol{h}\|^{2}}\left(\frac{v}{1-Z_{0}}\right) d v \\
& =\frac{1}{p_{\mathrm{M}}} \mathbb{E}_{Z_{0}} \frac{1}{(1-Z)^{N}} \int_{\gamma_{t h} r^{\alpha}}^{\infty} e^{-\left(w r^{-\alpha}+\frac{1}{1-Z_{0}}\right) v} \frac{v^{N-1}}{\Gamma(N)} d v \\
& =\frac{1}{p_{M}} \mathbb{E}_{Z_{0}} \frac{\gamma_{u}\left(N, \frac{\gamma_{t h} r^{\alpha}}{1-Z_{0}}+w \gamma_{t h}\right)}{\left(1+w r^{-\alpha}\left(1-Z_{0}\right)\right)^{N}} .
\end{aligned}
$$

Substituting (24) into (23) completes the proof.

For RVQ, as the previous sections we apply Jensen's inequality followed by straightforward mathematical manipulations to reach the following upper-bound

$$
R_{\mathrm{M}}^{u}=p_{\mathrm{M}} \log \left(1+\frac{\mathbb{E}_{Z}\left[(1-Z)^{2} \gamma_{u}\left(N+1, \frac{\gamma_{t h} r^{\alpha}}{1-Z}\right)\right]}{(1-\tilde{\delta}) p_{\mathrm{M}}^{\frac{\alpha}{2}+1}} \hat{C}(\alpha)\right)
$$

Let $\dot{p}_{\mathrm{M}}$ be the derivative of $p_{\mathrm{M}}$ with respect to $\gamma_{t h}$ :

$$
\dot{p}_{\mathrm{M}}=r^{\alpha} \mathbb{E}_{Z}\left[\frac{1}{1-Z} \mathrm{f}_{\|\boldsymbol{h}\|^{2}}\left(\frac{\gamma_{t h} r^{\alpha}}{1-Z}\right)\right]
$$

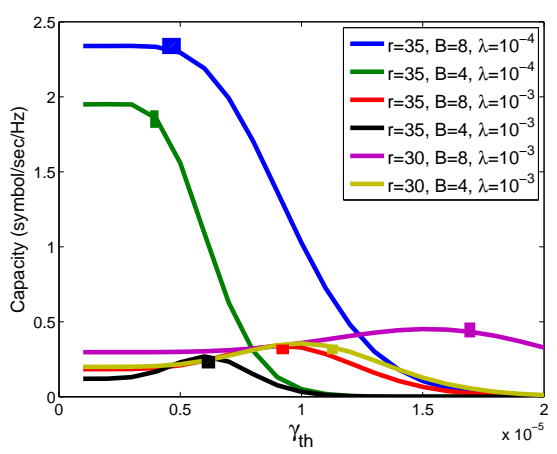

Fig. 3. Achievable capacity of MCQI-based scheduling vs. $\gamma_{t h}$ for $\alpha=4$ and $N=5$.

Thus, the best threshold value can be estimated by solving the following equation for $\gamma_{t h}$

$$
\begin{gathered}
\log \left(1+\frac{\mathbb{E}_{Z}\left[(1-Z)^{2} \gamma_{u}\left(N+1, \frac{\gamma_{t h} r^{\alpha}}{1-Z}\right)\right]}{(1-\tilde{\delta}) p_{\mathrm{M}}^{\frac{\alpha}{2}+1}} \hat{C}(\alpha)\right) \\
=\frac{\left(\frac{\alpha}{2}+1\right) \mathbb{E}_{Z}\left[(1-Z)^{2} \gamma_{u}\left(N+1, \frac{\gamma_{t h} r^{\alpha}}{1-Z}\right)\right]+N p_{\mathrm{M}} \gamma_{t h} r^{\alpha}}{\frac{1-\tilde{\delta}}{\hat{C}(\alpha)} p_{\mathrm{M}}^{\frac{\alpha}{2}+1}+\gamma_{u}\left(N+1, \gamma_{t h} r^{\alpha}\right)} .
\end{gathered}
$$

Fig. 3 presents the achieved capacity as in (21) vs. $\gamma_{t h}$ for several values of density and feedback capacity. We also show the obtained estimate of $\gamma_{t h}$ in Fig. 3 obtained according to equation (26). As it is seen the estimated values of $\gamma_{t h}$ are accurate enough to predict the best threshold value resulting in the maximum achievable capacity.

Note that compared to Fig. 2, here we observe that the capacity is much more sensitive to the value of $B$ for different values of $\gamma_{t h}$ and density. Moreover, as the case of CQI-based scheduling, here we observe that selecting other values for the threshold rather than the optimal obtained may result in a very low achievable capacity.

\section{Simulation Results}

In this section we investigate the accuracy of the analytical results in this paper through simulations. Simulations are conducted using Monte Carlo method. We also set $\alpha=4$, $r=30$ meters, and $N=4$.

In Fig. 4 and Fig. 5 we show the accuracy of the analysis for the cases of $B=4$ bits and $B=8$ bits, respectively. Here we set parameter $\gamma_{t h}=10^{-6}$ (CQI- and MCQI-based scheduling), and activity factor $p=0.6$ (Aloha). These illustrations confirms the accuracy of the analysis presented in this paper.

The accuracy of the obtained upper-bounds on the achievable capacity are confirmed in Figs. 1, 2, and 3. We therefore, in the following use the results provided on the selection of best activity factor based on Eq. (12) in the case of Aloha system, $\gamma_{t h}$ from (19) in the case of QCI-based scheduling, and finally $\gamma_{t h}$ from (26) in the case of MQCI-based scheduling to compare the performance of these systems with each other. 


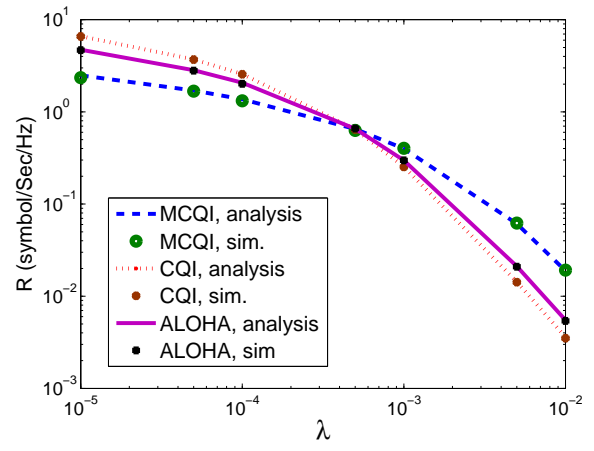

Fig. 4. Achieved capacity versus density, $\lambda$ for $B=4$ bits, $\gamma_{t h}=10^{-6}$ (CQI- and MCQI-based scheduling), and $p=0.6$ (Aloha).

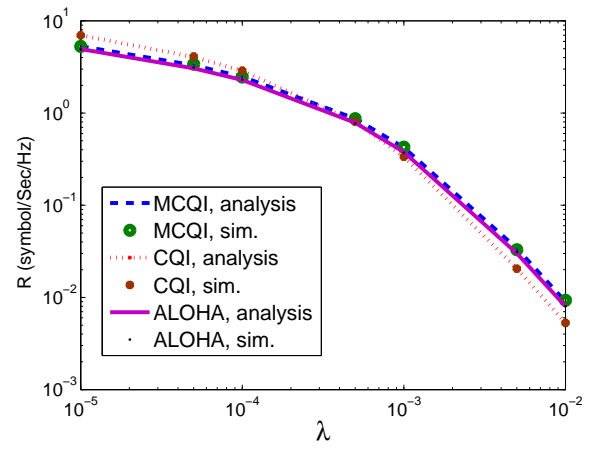

Fig. 5. Achievable capacity versus density $\lambda$, for $B=8$ bits, $\gamma_{t h}=10^{-6}$ (CQI- and MCQI-based scheduling), and $p=0.6$ (Aloha).

The results are reported in Fig. 6. As it is seen, the capacity under all of these schemes is logarithmically reduced by increasing $\lambda$. More importantly, MCQI-based scheduling outperforms both Aloha and CQI-based scheduling. The performance boost is higher in the case of a small feedback capacity $(B=4)$, while for the cases where the feedback capacity is high $(B=$ 8 ) the resultant performance boost is not substantial. It is also indicated that for $B=4$, Aloha outperforms the CQI-based scheduling. Finally, Fig. 6 reveals that MCQI-based scheduling demonstrates robustness against low feedback capacity, which is an important practical characteristic.

\section{CONCLUSION}

We investigated the capacity of several important scheduling schemes in limited-feedback MISO ad hoc networks. We mainly focused on Aloha, (CQI)-based scheduling, and MCQIbased scheduling. Stochastic geometry means adopted to derive the corresponding capacity of each scheme. Simulations proved the accuracy of our analysis. We furthermore observed that that MCQI-based scheduling improved the capacity compared to CQI-based scheduling and Aloha.

\section{REFERENCES}

[1] A. Asadi et al., "A survey on device-to-device communication in cellular networks," IEEE Comm. Surveys \& Tutorials, vol. 16, no. 4, pp. 18011819, 2014.

[2] Y. George et al., "The ergodic rate density of MIMO ad-hoc networks," IEEE 28-th Convention of Electrical and Electronics Engineers in Israel, 2014.

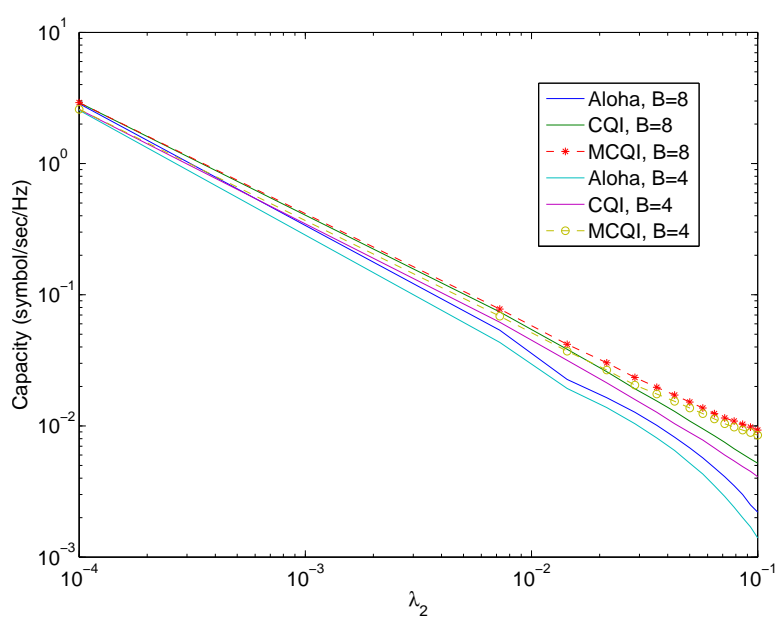

Fig. 6. The achievable capacity in Aloha, CQI- and MCQI-based scheduling $v s$. density for the case that the scheduling parameters are are optimized.

[3] K. Huang et al., "Spatial interference cancellation for multiantenna mobile ad hoc networks," IEEE Trans. Inf. Theory, vol. 58, no. 3, pp. 1660-1676, Mrc. 2012.

[4] R. Vaze and R. W. H. Jr., "Transmission capacity of ad-hoc networks with multiple antennas using transmit stream adaptation and interference cancellation," IEEE Trans. Inf. Theory, vol. 58, no. 2, pp. 780-792, Feb. 2012.

[5] N. Jindal et al., "Multi-antenna communication in ad hoc networks: Achieving MIMO gains with SIMO transmission," IEEE Trans. on Comm., vol. 59, no. 2, pp. 529-540, Feb. 2011.

[6] K. K. Mukkavilli et al., "On beamforming with finite rate feedback in multiple-antenna systems," IEEE Trans. Inf. Theory, vol. 49, no. 10, pp. 2562-2579, Oct. 2003.

[7] Y. Wu et al., "MIMO beamforming with quantized feedback in ad hoc networks: Transmission capacity analysis," ASILOMAR, pp. 1582-1587, Nov. 2010.

[8] M. Kountouris and J. G. Andrews, "Downlink sdma with limited feedback in interference-limited wireless networks," IEEE Trans. Wireless. Comm., vol. 11, no. 8, pp. 2730-2741, Aug. 2012.

[9] J. Park and R. W. H. Jr., "Multiple-antenna transmission with limited feedback in device-to-device networks," to appear in IEEE Wireless Comm. Letters, 2016.

[10] F. Baccelli et al., "An ALOHA protocol for multihop mobile wireless networks," IEEE Trans. Information Theory, vol. 52, no. 2, pp. 421-436, Feb. 2006.

[11] S. P. Weber et al., "The effect of fading, channel inversion, and threshold scheduling on ad hoc networks," IEEE Trans. Information Theory, vol. 53, no. 11, pp. 4127-4149, Nov. 2007.

[12] M. Haenggi and R. K. Ganti, "Interference in large wireless networks," Foundations and Trends in Networking, vol. 3, no. 2, 2008, Available at http://www.nd.edu/ mhaenggi/pubs/now.pdf.

[13] N. Jindal, "MIMO broadcast channels with finite-rate feedback," IEEE Trans. Inf. Theory, vol. 52, no. 11, pp. 5045-5060, Nov. 2006.

[14] M. Haenggi and other, "Stochastic geometry and random graphs for the analysis and design of wireless networks," IEEE JSAC, vol. 27, no. 7, pp. 1029-1046, Sep. 2009.

[15] K. Hamdi, "Capacity of MRC on correlated rician fading channels," IEEE Trans. Comm., vol. 56, no. 5, pp. 708-711, May 2008. 